
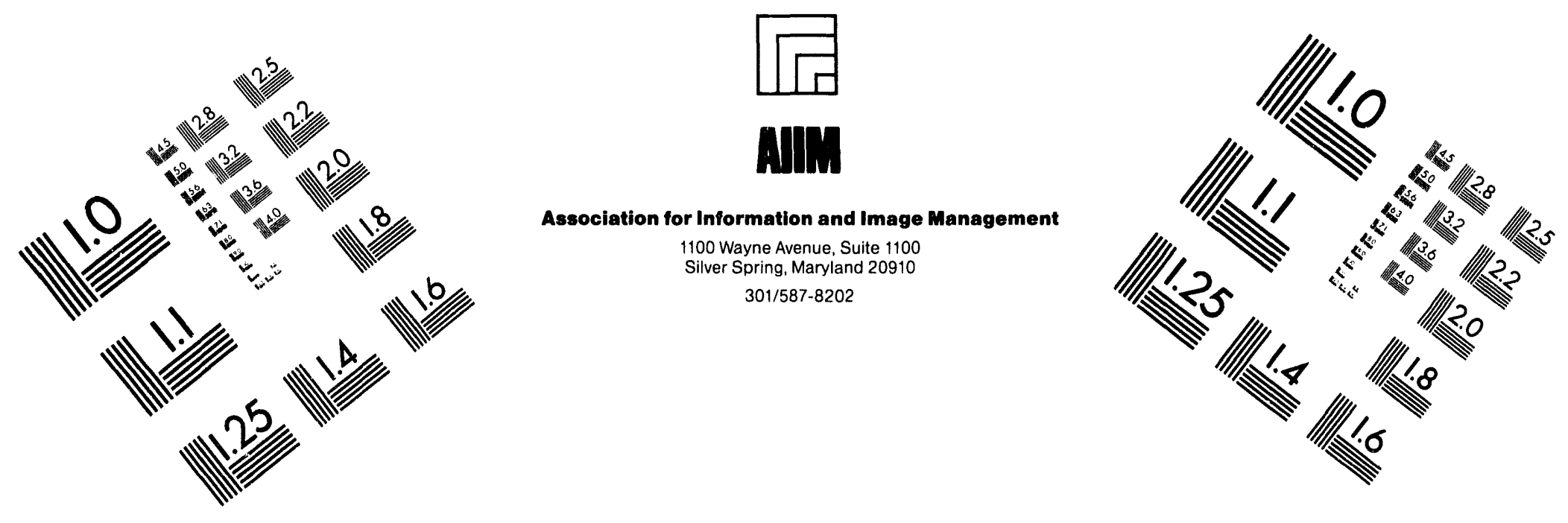

\title{
Centimeter
}

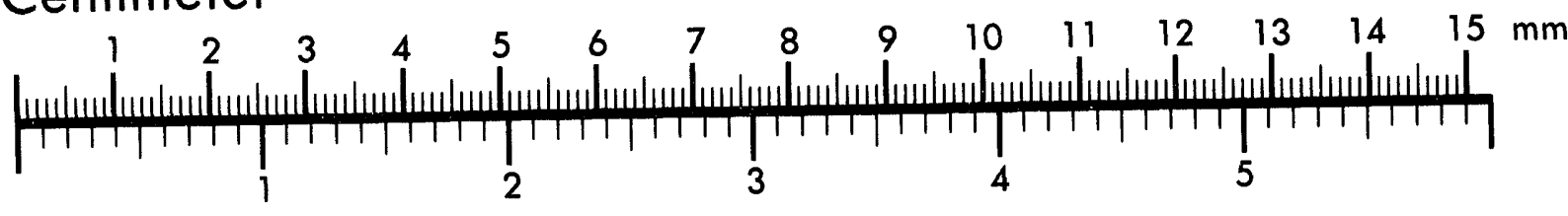
Inches
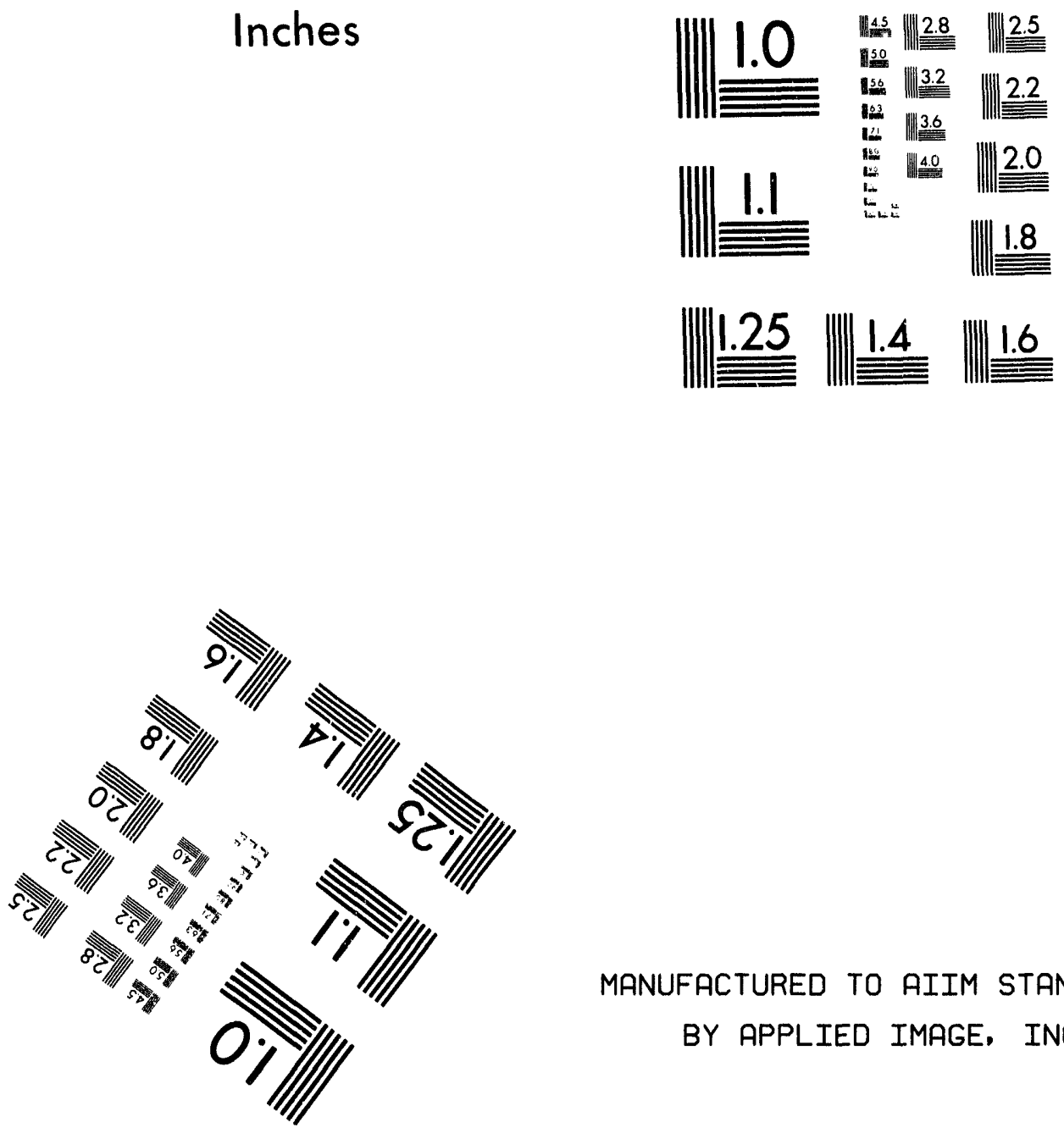

MANUFACTURED TO AIIM STANDARDS

BY APPLIED IMAGE, INC.

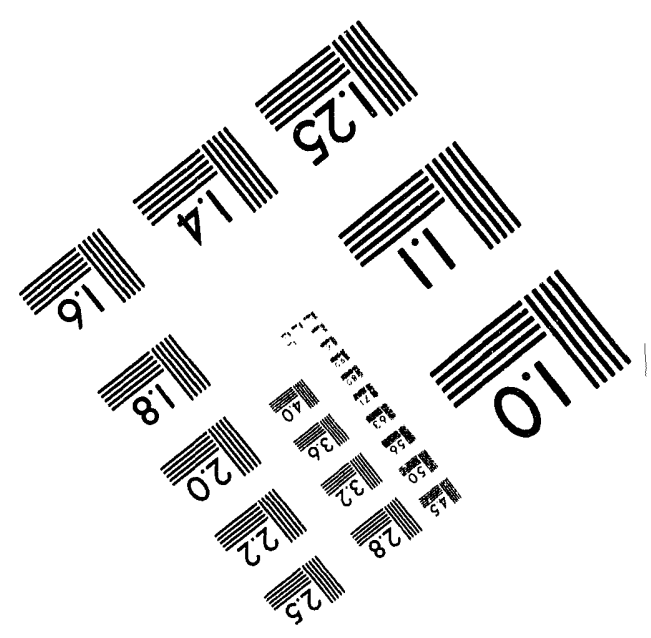



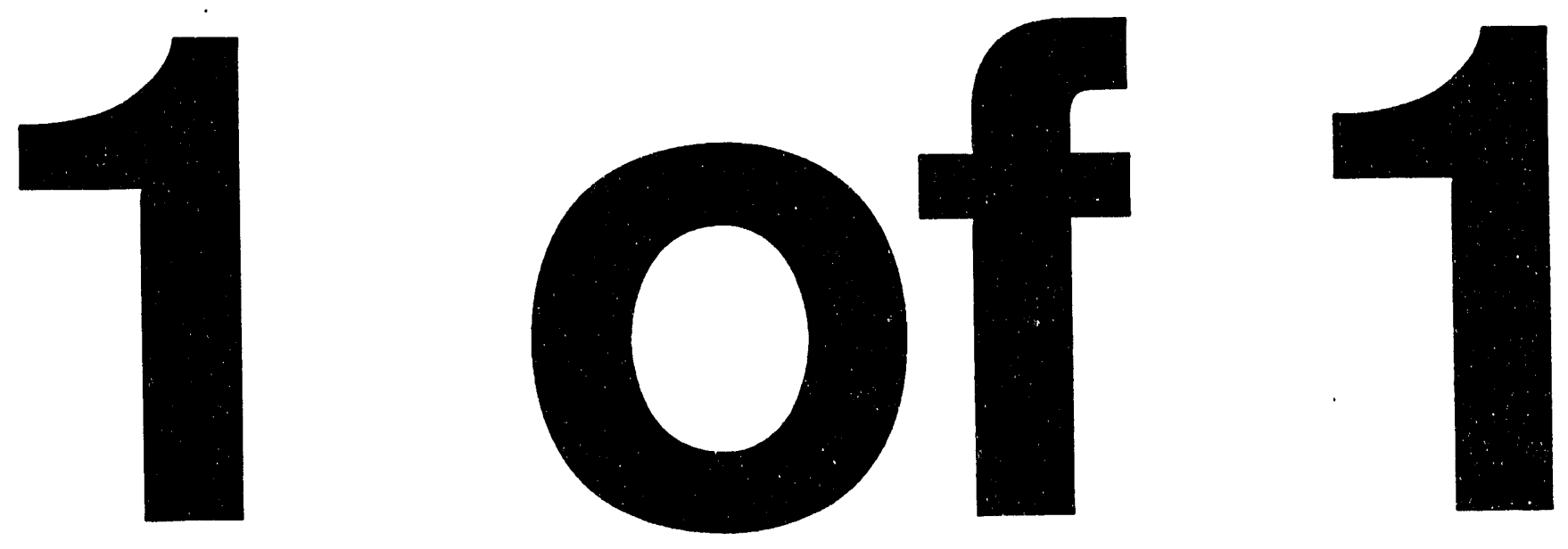


\section{Fragmentation of Hot Nuclear Systems Formed in Xe-Induced Reactions at Ebeam-60 MeV/nucleon}

Presented at the 9th High-Energy Heavy-Ion Study, Berkeley, CA 94720

October 25-29, 1993

W. Skulski, K. Tso, N. Colonna, G. J. Wozniak, L.G. Moretto,

D.R. Bowman, M. Chartier, C.K. Gelbke, W.C. Hsi, M.A. Lisa, W.G. Lynch, G.F. Peaslee, L. Phair, C. Schwarz, and M.B. Tsang

Lawrence Berkeley Laboratory, University of California, Berkeley, CA 94720, USA

October 1993

This work was supported in part by the Director, Office of Energy Research, Division of Nuclear Physics of the Office of High Energy and Nuclear Physics of the U.S. Department of Energy under Contract DE-AC0376 SF00098.
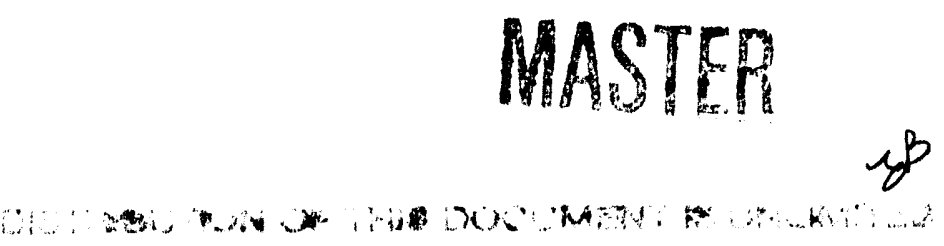
Fragmentation of Hot Nuclear Systems Formed in Xe-Induced Reactions at $\mathbf{E}_{\text {beam }}=60 \mathrm{MeV} /$ nucleon\#

W. Skulski, ${ }^{*}$ K. Tso, N. Colonna, G. J. Wozniak, L. G. Moretto, D. R. Bowman, M. Chartier ${ }^{+}$, C. K. Gelbke ${ }^{+}$, W. C. Hsit , M. A. Lisa ${ }^{+\$}$, W. G. Lynch , G. F. Peaslec+@, L. Phair ${ }^{+}$, C. Schwarz ${ }^{+}$, and M. B. Tsang ${ }^{+}$

\section{Introduction}

Multifragment disintegration of highly excited nuclear systems is the subject of much current interest, both experimentally and theoretically (see (1) and references therein). In theoretical calculations, one can specify the range of impact parameters of the reaction or its "centrality". In experimental studies however, the impact parameter is not measured directly. Therefore, one typically uses the light charged particle multiplicity as a measure of the centrality of the collision.2,3) Recently, a study of the $\mathrm{Xe}+\mathrm{Bi}$ reaction at $28 \mathrm{MeV} /$ nucleon indicated that a binary reaction mechanism persists even for high particle multiplicity and/or large intermediate mass fragment (IMF) multiplicity. ${ }^{4}$ ) Thus, it is not clear if central collisions are well characterized by the sole requirement of high particle multiplicity. The aim of the present paper was to investigate reactions of Xe with a range of targets to see, to what extent a binary reaction pattern persists at $60 \mathrm{MeV} /$ nucleon.

\section{Experimental procedure}

The experiment was performed at the K1200 Cyclotron of the National Superconducting Cyclotron Laboratory at Michigan State University. A $60 \mathrm{MeV} /$ nucleon ${ }^{129} \mathrm{Xe}$ beam bombarded targets of ${ }^{27} \mathrm{Al}$, nat $\mathrm{Cu},{ }^{89} \mathrm{Y},{ }^{165} \mathrm{Ho}$ and ${ }^{197} \mathrm{Au}$ of thickness $2.07,2.0,1.0,2.0$ and 1.3 $\mathrm{mg} / \mathrm{cm}^{2}$, respectively. The detection system subtended angles from 20 to $160^{\circ}$ with respect to the beam axis and had a geometric acceptance of $\sim 88 \%$ of $4 \pi$. At forward angles $\left(2^{\circ}-16^{\circ}\right)$, fragments $(Z=1-54)$ were detected in a 16-telescope $\operatorname{Si}(300 \mu \mathrm{m})-\operatorname{Si}(5 \mathrm{~mm})$-Plastic $(7.6 \mathrm{~cm})$ array ${ }^{5)}$ with good energy and position resolution. The geometrical efficiency of the forward array was about $64 \%$. Individual elements were resolved for $Z=1$ to 54 , when counting statistics allowed. Representative detection thresholds in the forward array were 13, 21 and $27 \mathrm{MeV} / \mathrm{u}$ for fragments of $\mathrm{Z}=8,20$ and 54, respectively. Energy and position calibrations were performed by utilizing analog beams of $\mathrm{q} / \mathrm{A}=1 / 6\left[\mathrm{D}^{4} \mathrm{He}^{+}, 6 \mathrm{Li}^{+}, 12 \mathrm{C}^{2+}, 18 \mathrm{O}^{3+}\right]$ at 22 $\mathrm{MeV} /$ nucleon, and two "cocktail" beams $\left.{ }^{6}\right)$ at $60 \mathrm{MeV} /$ nucleon: $\left[{ }^{30} \mathrm{Si},{ }^{60} \mathrm{Ni}, 90 \mathrm{Zr}\right.$ ] and [ ${ }^{43} \mathrm{Ca}$, ${ }^{86} \mathrm{Rb},{ }^{129} \mathrm{Xe}$ ]. (A "cocktail" beam consists of 3 different ion beams with nearly identical charge to mass ratios. ) These low intensity beams were swept directly across each of the array telescopes. These data were used to measure the nonuniformity in the $300 \mu \mathrm{m} \Delta \mathrm{E}$ detector thickness and corrections were made off-line. The pulse-height defect was measured and corrected for according to ref. (7). The overall energy calibration was accurate to about $1 \%$ and the position calibration to within $1.5 \mathrm{~mm}$.

At larger angles $\left(16^{\circ}-160^{\circ}\right)$, light charged particles and fragments $(Z=1-35)$ were detected in the MSU Miniball $\left.{ }^{8}\right)$ consisting of 171 fast plastic $(40 \mu \mathrm{m})-\mathrm{CsI}(2 \mathrm{~cm})$ phoswhich detectors. The most forward-angle ring and four detectors of the second ring of the Miniball were removed to accommodate the forward-angle $\mathrm{Si}$ array. Representative detection thresholds were 2,3 , and $4 \mathrm{MeV} / \mathrm{u}$ for $\mathrm{Z}=3,10$, and 18 fragments. For $\mathrm{H}$ and $\mathrm{He}$, individual isotopes could be resolved. For heavier elements, only elemental resolution was achieved. The energy calibration was obtained by scaling the previous calibration ${ }^{9}$ ) to hydrogen punchthrough points in every detector. This procedure was checked for a subset of 8 Miniball 
detectors that were extensively calibrated by sweeping the $q / A=1 / 6$ beams across the face of the detectors. For this limited subset of detectors, a satisfactory agreement with the existing calibration was found.

\section{Results}

\section{Global reaction patterns}

As the excitation energy of the heavy reaction products is increased, one expects the number of evaporated neutrons and light charged particles to increase. Thus, many groups have used $4 \pi$ neutron or chargei particle detectors to determine the extent to which the kinetic energy available in the entrance channel is thermalized. In fact, recent measurements ${ }^{4}$ ) that combine both detection techniques have shown a strong correlation between the measured neutron and charged particle multiplicities. In our measurements, we utilize the total charged particle multiplicity $M$ which consists of the measured number of light charged particles (LCPs) and intermediate mass fragments (IMFs). Low $M$ values are characteristic of low-dissipation, peripheral events, while high $M$ is associated with more violent, central collisions. One should note, that for light targets $(\mathrm{Al}, \mathrm{Cu})$ the present experiment does not detect the most peripheral events with good efficiency due to the grazing angle falling below the minimum detection angle of the forward array. In this case a PLF can be detected in the forward array only if it is deflected to $\theta>2^{\circ}$ due to a sequential decay. This introduces a certain bias against large impact parameter events. For heavier targets (Y, Ho, $\mathrm{Au}$ ) events from a large range of impact parameters can be observed.

For the ideal $4 \pi$ detection system with $100 \%$ efficiency, one would expect to see constant value of the total detected kinetic energy, independent of the degree of dissipation, except for small variations due to different Q-values of the different reaction channels. For the actual detection system, the total detected energy is smaller than the idealized limit. First, neutrons are not measured by the present experimental setup. Second, some charged particles may not be observed due to thresholds and dead regions between the detectors. Figure 1 shows a comparison of the total detected charge, kinetic energy and linear momentum versus the total number of detected particles $\mathrm{M}$ for five targets. For the lightest target ${ }^{27} \mathrm{Al}$, the total charge distribution has two peaks. For the upper peak, $90 \%$ of the total charge of the system is detected $\left(Z_{\text {total }}=\sim 50\right)$, whereas for the lower peak, only a small percentage is detected $\left(Z_{\text {total }}=\sim 10\right)$. The lower peak corresponds to the situation when the heavy projectile-like fragment (PLF) is not detected due either to it being emitted into an angle smaller than $2^{\circ}$ or the dead region between telescopes. When the heavy forward-going PLF is not detected, one sees only light particles emitted from the target and projectile-like residues. For heavier targets, one observes two branches (e.g., see the Au target). For small values of $M$, the upper branch starts at the projectile $\mathrm{Z}$-value. As $\mathrm{M}$ increases, this branch rises slowly, and then joins the rapidly rising lower branch. For the most central collisions, the total detected charge is greater than the projectile charge. For heavy targets, the two-branch region can be associated with peripheral collisions: the lower branch arises when the forward-going PLF is missed and only LCPs have been detected; whereas the upper branch results when both are detected. For peripheral collisions, one does not detect the total charge of the system because the slow moving target-like fragment (TLF) is below the detector thresholds. As the impact parameter decreases, the TLF becomes more excited and emits increasing numbers of light charged particles causing the lower branch to increase until the two branches merge. For the most central collisions, the system presumably breaks into many small fragments and no large projectile-like or target-like fragment remain. A very similar pattern is observed in plots 
of the total kinetic energy and the total linear momentum detected versus the total multiplicity (see Figure 1).

$60.0 \mathrm{MeV} /$ nucleon ${ }^{120} \mathrm{Xe}+\mathrm{X}$

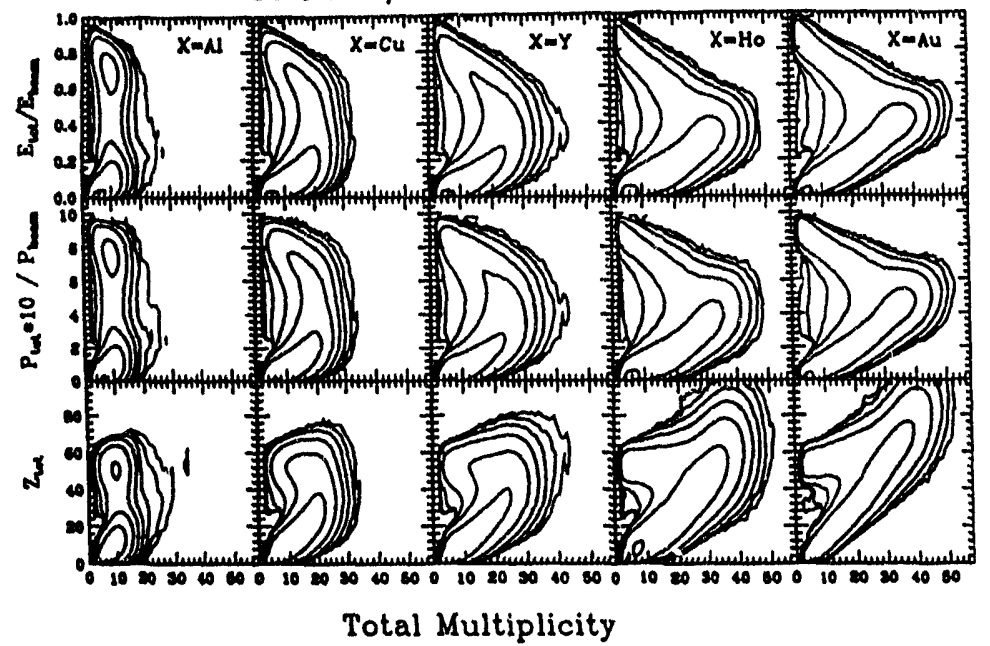

Fig. 1. For each event, the detected total energy (upper row), total detected parallel momentum (second row) and total detected charge (third row) are plotted vs. total charged particle multiplicity, for the $60 \mathrm{MeV} / \mathrm{u}{ }^{129} \mathrm{Xe}+{ }^{27} \mathrm{Al}$, nat $\mathrm{Cu},{ }^{89} \mathrm{Y},{ }^{165} \mathrm{Ho}$ and ${ }^{197} \mathrm{Au}$ reactions.

The two branches in Fig. 1 should have roughly the same slope as a function of $M$, unless the size of the largest fragment decreases with $M$, in which case the branches should merge for high values of $M$. This is indeed the case, as shown in Figure 2, in which the mass of the largest fragment detected in every event is plotted as a function of $M$. (The mass was inferred from the measured charge according to ref. (10).) Two branches can be clearly seen in case of heavier targets like $\mathrm{Ho}$ and $\mathrm{Au}$, while for $\mathrm{Al}$ the distribution resembles the previous figure. A clear anti-correlation of the size of the largest detected fragment with $M$ is observed for all targets except Al. The parallel to the beam velocity component of the largest detected fragment is roughly constant as a function of multiplicity, as shown in Figure 3. For low and intermediate multiplicity, the largest fragments are the dominant, "leading" ones, and they carry memory about the projectile. The leading fragments contribute roughly constant energy per nucleon and constant momentum per nucleon to the measured total energy and total momentum in Fig. 1. At the same time, there is a big difference in mass between the biggest and the next biggest detected fragment, see Figure 2.

Projectile-like fragments can be produced following substantial energy dissipation, as indicated by the high charged-particle multiplicity values. The dissipated energy is being transferred to the collision products at the expense of the initial kinetic energy present in the entrance channel. This is shown in Figure 3, in which the parallel velocity of the largest fragment is plotted as a function of $M$. For low dissipation (low multiplicity values) the PLFs have almost the beam velocity, while for large dissipation (large $M$-values) the decrease in the PLF velocity is substantial. This decrease corresponds to a large energy missing from the entrance channel. For example, if a fragment of $Z=35, A=80$ is slowed to about $90 \%$ of the beam velocity, it loses about $870 \mathrm{MeV}$. It is also interesting to note, that for targets heavier than Al for high values of $\mathrm{M}$, a low-velocity target-like component gradually builds up in the velocity spectra in Figure 3. 


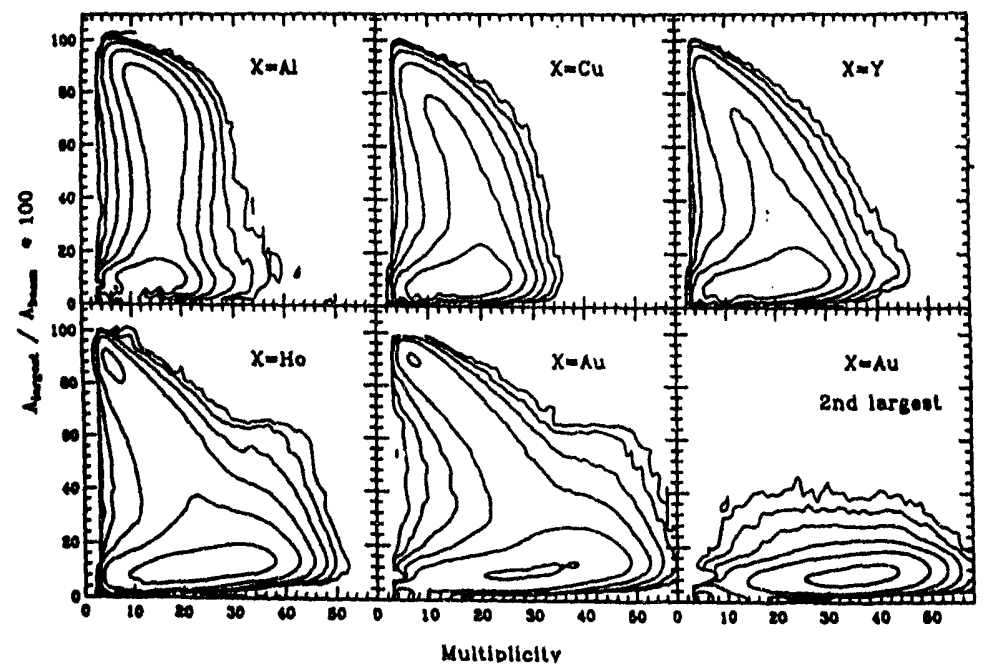

Fig. 2. For each event, the mass of the largest detected fragment is plotted vs. total charged particle multiplicity, for the $60 \mathrm{MeV} / \mathrm{u}{ }^{129} \mathrm{Xe}+{ }^{27} \mathrm{Al}$, nat $\mathrm{Cu},{ }^{89} \mathrm{Y},{ }^{165} \mathrm{Ho}$ and ${ }^{197} \mathrm{Au}$ reactions.
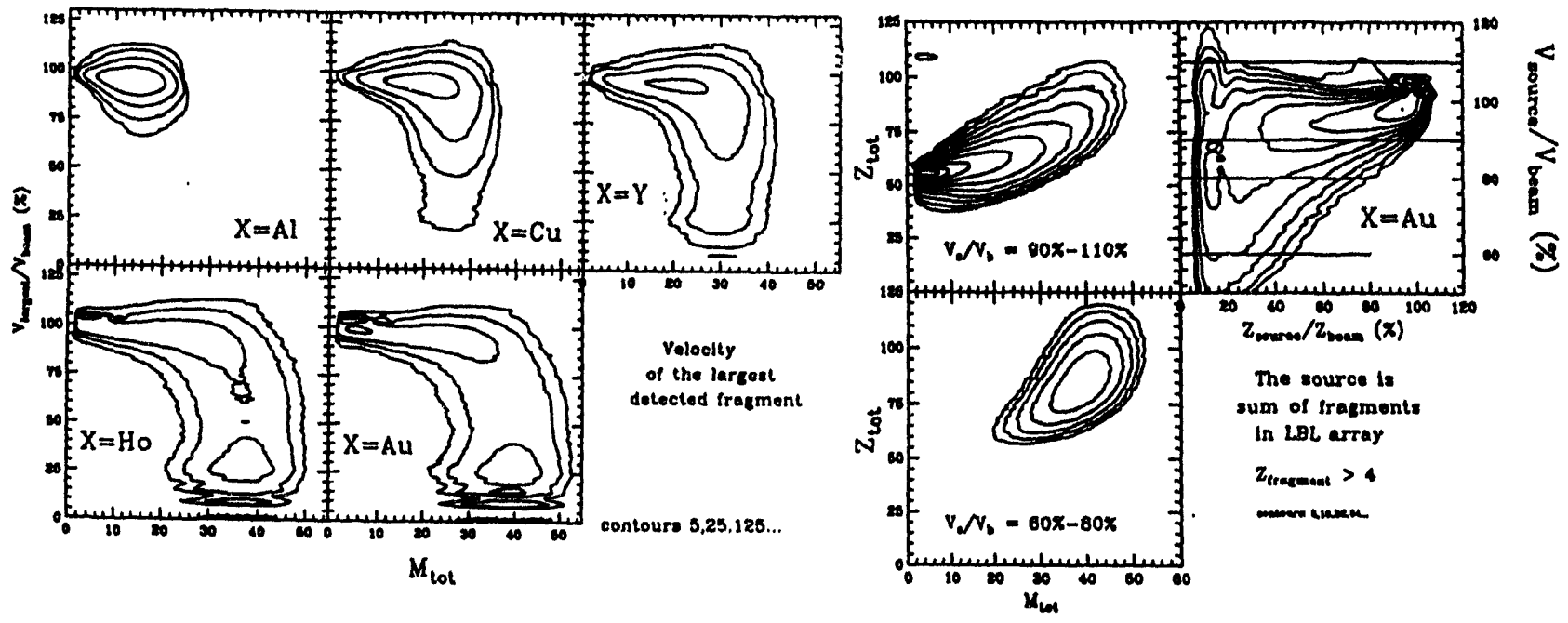

Fig. 3. For each event, the velocity of the largest detected fragment is plotted against the total charged particle multiplicity $M$ for the $60 \mathrm{MeV} / \mathrm{u}{ }^{129} \mathrm{Xe}+{ }^{27} \mathrm{Al}$, nat $\mathrm{Cu}$, ${ }^{89} \mathrm{Y},{ }^{165} \mathrm{Ho}$ and ${ }^{197} \mathrm{Au}$ reactions. Velocity is relative to beam velocity.

Fig. 4. For $60 \mathrm{MeV} /$ nucleon ${ }^{129} \mathrm{Xe}+{ }^{197} \mathrm{~A} \mathrm{u}$ reaction. Upper right panel: Contour plot of the mean parallel velocity of the PLF source versus the size of the source (see text). The left-hand panels: The distributions of the total detected charge $Z_{t o t a l}$ versus multiplicity, gated on two ranges of the PLF source velocity: $90 \%-110 \%$ and $60 \%-80 \%$ of $\mathrm{V}_{\text {beam. }}$. More dissipative events correspond to slower PLF sources, as shown by the increased multiplicity and $Z_{\text {total }}$.

\section{Primary PLF fragments}

As the energy dissipation in the reaction increases, as reflected by the increasing multiplicity, the excitation of the forward-going primary PLF also increases. At large 
excitation energies, the primary PLF can decay into a several secondary fragments. With our forward array we can detect most of them. During the off-line analysis, we reconstructed the primary PLF "source". We defined a "primary source" as being the sum of all fragments detected in the forward array, $\mathrm{Z}_{\text {fragment }}>4.1$ ) When discussing "PLF sources", only "completely" measured events will be considered, defined as those with total detected energy of at least $50 \%$ of the beam energy (see the first row in Figure 1). This $50 \%$ cutoff serves as a convenient discrimination against incompletely measured events, in which the PLF was not detected due to less than $100 \%$ detection efficiency.

In Figure 4 the parallel velocity of the reconstructed PLF source is plotted versus its reconstructed charge (upper-right panel). The triangular pattern of the charge-velocity contour plots is similar to those previously reported in the La-induced reactions between 35 and 55 $\mathrm{MeV} /$ nucleon. 1) This pattern was previously interpreted as a result of the incomplete fusion followed by extensive particle evaporation, see (1) for details. One of the conclusions of the previous study was that slower PLF sources correspond to increased energy dissipation. One can test this by looking at the total detected charge and/or multiplicity in coincidence with PLF "sources" moving with different velocities. Both the total detected charge and total multiplicity are increased on the average, when the gate is set on the slower-moving PLF source, see the left-hand side of Figure 4. The increase is due to higher degree of dissipation, what corroborates the conclusions of ref. (1). One should note a conspicuous absence of peripheral collisions in the lower left panel of Figure 4.

\section{Determination of the reaction plane}

One of the key features of a binary PLF-TLF reaction is a strong kinematical correlation between the primary projectile-like and target-like fragments. Even though the present experiment did not detect target-like fragments with good efficiency due to detection thresholds in the Miniball, the PLF-TLF correlation can be explored indirectly through the LCP and light fragment emission patterns. Namely, the primary PLF source (for which the detection efficiency is almost complete) can be utilized to determine the reaction plane. The emission pattern in the Miniball can then be examined to determine the correlation with the primary PLF, when viewed relative to the reaction plane. Figure 5 shows the distribution of the relative azimuthal angle between the PLF and TLF sources. The azimuthal angle in the array $\phi_{P L F}$ is defined as that of the primary PLF source, while $\phi_{T L F}$ is the azimuthal angle of the average momentum of all particles detected in the Miniball (excluding punch-through $\mathrm{Z}=1$ particles). The relative azimuthal angle is the difference $\phi_{\text {Telative }}=\phi_{\mathrm{PLF}}-\phi_{\mathrm{TLF}}$. It shows a very distinct peak at $\phi_{\text {relative }}=180^{\circ}$, indicating a strong directional anticorrelation between the two sources. This anticorrelation is evidence for a binary reaction mechanism. The width of the peak is of the order of $60^{\circ}$ in case of $\mathrm{Al}$ target, and close to $100^{\circ}$ in case of Au target. For the $\mathrm{Au}$ target the width increases as a function of multiplicity. In $\mathrm{Al}$ case the width changes very little as a function of multiplicity, since mainly central collisions are detected in case of $\mathrm{Al}$ target, as discussed earlier.

\section{Summary and conclusions}

We have established that in $60 \mathrm{MeV} /$ nucleon ${ }^{129} \mathrm{Xe}+{ }^{27} \mathrm{Al}$, nat $\mathrm{Cu},{ }^{89} \mathrm{Y},{ }^{165} \mathrm{Ho}$ and ${ }^{197} \mathrm{Au}$ reactions a projectile-like fragment is formed, especially at low values of multiplicity. The velocity of the PLF $s$ close to the beam velocity even though its mass changes with multiplicity from close to the projectile mass (at low value of multiplicity) to a small fraction at high multiplicity values. The distributions of total detected energy, total momentum and total charge versus multiplicity show a characteristic two-branch pattern. At a given 
multiplicity, the upper branch is due to completely measured events, where the leading projectile-like fragment has been detected. The lower branch results, when the leading fragment is not detected, either due to the small grazing angle or to the incomplete efficiency of the forward array.

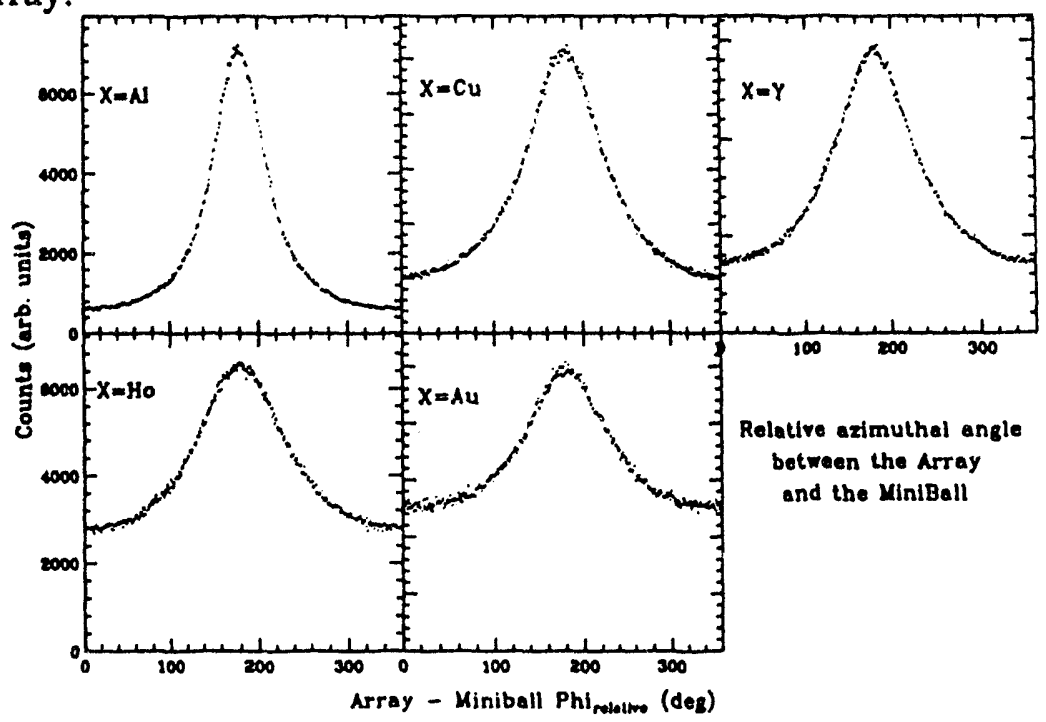

Fig. 5. The distribution of the relative azimuthal angle $\phi_{\text {relative }}$ between the PLF source in the forward array and the mean momentum vector in the Miniball.

For heavier targets (Au, Ho) there is also a region above roughly $\mathrm{M}=35$, where the two branches merge together and there seems to be no clearly defined leading fragment. Events above $\mathrm{M}=35$ therefore seem to be promising candidates for "central collisions", characterized by complete overlap of the projectile with the target. Even there, substantial fragments with parallel velocity component close to beam velocity were detected. Thus, a substantial fraction of highly dissipative events seems to be of a binary-like type.

The parallel velocity of the reconstructed PLF source was used to select events with varying degree of dissipation. More dissipative events were selected on the average in coincidence with slow sources, than the ones observed in coincidence with fast moving sources. A PLF source - TLF source directional anticorrelation was demonstrated.

\section{Foomotes and References}

\#This work was supported by the Dir., Office of Energy Research, Div, of Nuclear Physics, Office of High Energy and Nuclear Physics of U.S. DOE.

* On leave of absence from Heavy Ion Laboratory, Warsaw University, Poland

${ }^{+}$NSCL, Michigan State University

Spresent address: Lawrence Berkeley Laboratory, Berkeley, CA 94720

@ Present address: Hope College, Chemistry Dept., Holland, MI 49423

1) L.G.Moretto and G.J.Wozniak, Annual Review of Nucl. and Part. Physics 43 (1993) 379.

2) D. R. Bowman, et al., Phys. Rev. Lett. 67 (1991) 1527.

3) D. R. Bowman, et al., Phys. Rev. C46 (1992) 1834.

4) B. Lott, et al., Phys. Rev. Lett. 68 (1992) 3141.

5) W. L. Kehoe, et al., Nucl. Instr. Meth. A311 (1992) 258.

6) M. A. McMahan, et al., Nucl. Instr. Meth. A253 (1986) 1.

7) J. B. Moulton, et al., Nucl. Instr. Meth. 157 (1978) 325.

8) R. T. d. Souza, et al., Nucl. Instr. and Meth. A295 (1990) 109.

9) Y. D. Kim, et al., Phys. Rev. C45 (1992) 338.

10) R. J. Charity, et al., Nucl. Phys. A483 (1988) 371. 

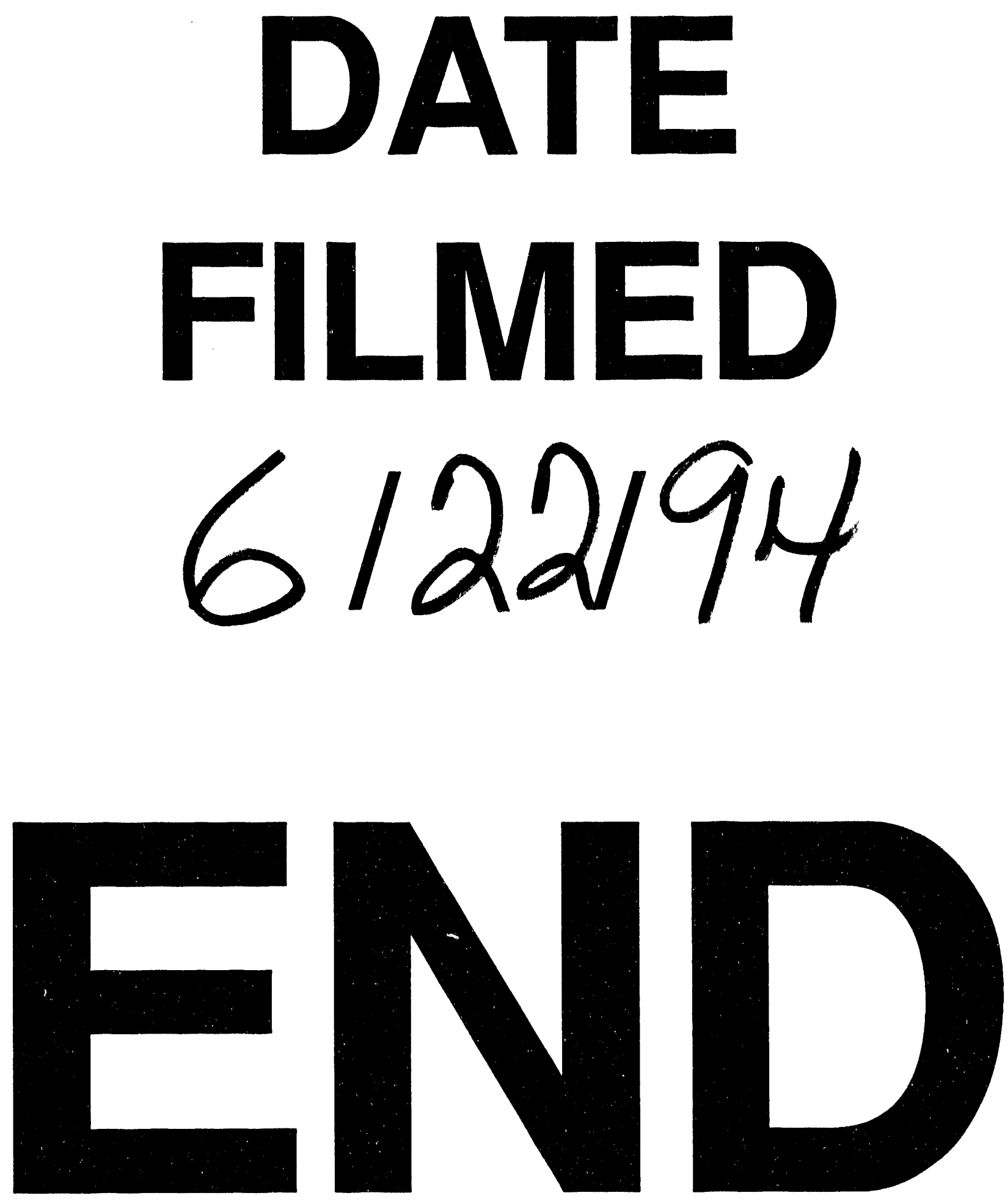


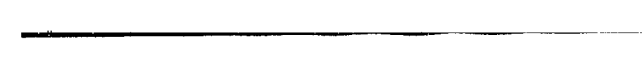

